\section{A Search for Low-energy Gamma-rays from the Quasar 3C286}

IN two experiments on the Ranger spacecraft, Metzger et al. ${ }^{1}$ have observed a flux of low-energy $\gamma$-rays at great distances from the Earth. Previous experiments on highaltitude balloons ${ }^{2,3}$ and on rockets ${ }^{4}$ had looked for, but failed to detect, an isotropic flux of $\gamma$-rays incident on the top of the Earth's atmosphere. These experiments used counters with little or no directional sensitivity, and their fajlure was due to the difficulty in distinguishing between an isotropic primary flux from the background of secondary $\gamma$-rays produced by cosmic rays in the atmosphere and in the apparatus itself. However, even in the presence of this background, a counter with directional sensitivity can detect $\gamma$-rays from point sources by measuring increases in the total flux in particular directions in the sky. We report here the results of observations made at balloon altitude on the quasar $3 C 286$.

The detector used in this experiment was a 2 -in. diam. $\times$ 2 -in. high sodium iodide scintillation erystal; it was surrounded by a collimator with an opening angle of $\pm 5^{\circ}$. The telescope was carried to an altitude of 100,000 ft. by balloon on October 28,1964 . During the flight the attitude of the telescope was controlled from the ground by radio-command. Pulse height spectra were recorded from the direction of $3 C 286$ and from neighbouring directions; the spectra were taken frequently, and alternately from the source and from the background, to avoid systematic errors due to changes in the overall gain of the system. A caesium-137 source was carried on the telescope to provide a calibration during the flight. The energy resolution was $\sim 0.5 \mathrm{MeV}$.

The combined spectrum recorded from the direction of $3 C 286$, with the background subtracted, is shown in Fig. 1. The spectrum is not significantly different from zero at any energy.

This result sets upper limits to the flux of $\gamma$-rays from several possible processes on $3 C 286$. In each case, the 95 per cent confidence limit is given. No allowance has been made for a possible red-shift of $3 C 286$; the results may need recalculating when the red-shift is oventually measured, but the changes in the limits will probably be small.

(1) Electron-positron annihilation. The limit to the flux of $0.51-\mathrm{MeV} \gamma$-rays is 0.04 photons $/ \mathrm{cm}^{2}$ sec at the top of the atmosphere.

(2) Neutron capture in hydrogen. The limit to the flux of $2.2-\mathrm{MeV} \gamma$-rays is 0.13 photons $/ \mathrm{cm}^{2}$ sec.

(3) Interaction of high-energy electrons with optical photons through the inverse Compton effect. Ginzburg et al..$^{5}$ have considered this process for the quasar $3 C 273$, the distance of which is already known. They have

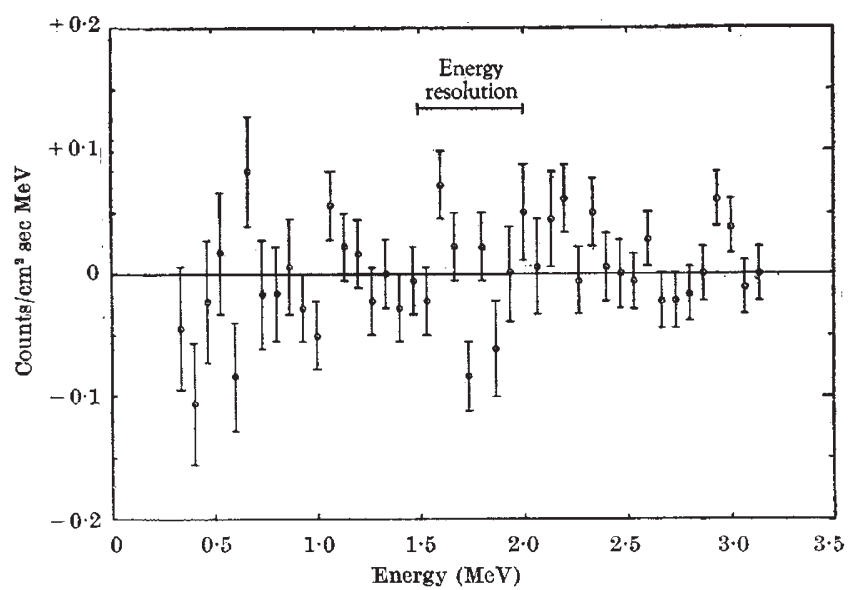

Fig. 1. Pulse height spectrum received from the direction of $3 C 286$ with the background from neighbouring directions subtracted. Error equal to one standard deviation are shown for each point shown that if the optical continuum is due to the synchro. tron radiation of high-energy electrons, then the same electrons should produce low-energy $\gamma$-rays in collisions with optical photons. If the optical radiation has a spectrum of the form:

$$
I(\nu) \mathrm{d} \nu=I_{0} \nu^{-a+1} \mathrm{~d} \nu
$$

then the $\gamma$-ray spectrum has the form:

$$
F(E) \mathrm{d} E=F_{0} E^{-\alpha} \mathrm{d} E
$$

The optical spectrum from $3 C 286$ is flat ${ }^{6}$, so the $\gamma$-ray spectrum should have $\alpha=1$. The experiment described here sets a limit to $F_{0}$ of $5 \cdot 5 \times 10^{-2}$ photons $/ \mathrm{cm}^{2} \mathrm{sec}$.

The results of Metzger et al. indicate a primary flux with a spectrum of the form:

$$
N(E) \mathrm{d} E=N_{0} E^{-2} \mathrm{~d} E
$$

The flux at $1 \mathrm{MeV}$, integrated over all directions in space, is $4 \times 10^{-1}$ photons $/ \mathrm{cm}^{2}$ sec $\mathrm{MeV}$. If a similarly shaped spectrum is being emitted by $3 C 286$ the present experiment shows that the limit to the flux at $1 \mathrm{MeV}$ is $3.6 \times 10^{-2}$ photons $/ \mathrm{cm}^{2}$ see $\mathrm{MeV}$.

We thank Prof. C. F. Powell for the hospitality of his laboratory. We also thank many members of the laboratory for their assistance, especially Dr. K. G. Standing (now at the University of Manitoba), who initiated the experiment, and Mr. J. H. Davies, who organized the bailloon flight.

The telemetry system was designed and built at the Atomic Weapons Research Establishment, Aldermaston. One of us (D. J. A.) thanks the Department of Scientific and Industrial Research for a maintenance grant.

D. J. ADAMS

R. R. HILlier

A. F. JANES

H. H. Wills Physics Laboratory, University of Bristol.

${ }^{1}$ Metzger, A. E., Anderson, E. C., Van Dilla, M. A., and Arnold, J. R., Nature. 204, 766 (1964)

2 Jones, F. C., J. Geophys. Res., 66, 2029 (1961)

${ }^{3}$ Peterson, L. E., J. Geophys. Res., 68, 979 (1963).

- Northrop, J. A., and Hostetler, R. L., Bull. Amer. Phys. Soc., 6, 52 (1961). ${ }_{5}$ Ginzburg, V. L., Ozernoi, L. M., and Syrovatski, S. J., Doklady, 9, 3 (1964). ${ }^{8}$ Matthews, J. A., and Sanadage, A. R., A strophys. J., 138, 30 (1963).

\section{GEOPHYSICS}

\section{Continental Drift and the Rotation of Spain}

Sir EDward Bullard et al. ${ }^{1}$ have used a 'least squares' criterion for the geometrical fit of the continents around the Atlantic Ocean. They find that the best fits are obtained when the 500-fathom submarine contours are used to define the continental margins. When all the continents are fitted, it is found on fitting Africa to eastern North America that Africa overlaps the position of southern Spain. To avoid this, Bullard et al. rotate Spain, closing up the Bay of Biscay as previously suggested for other reasons by du Toit ${ }^{2}$ and Carey ${ }^{3}$. The appropriate part of the computer fit is shown in Fig. 1. It is clearly desirable to look for some independent evidence for the rotation of Spain.

Irving 4 has noted that there is some palaeomagnetie evidence for such a rotation. The argument is that the declinations of the directions of remanent magnetization for rocks of Permian and Triassic ages from Spain are different from those of rocks of the same ages from France, Germany, Britain, Norway and the U.S.S.R. The latter are all consistent, indicating that these regions all formed one unit. With the aid of some new results from the northern Pyrenees, this argument is followed up and the palaeo-declinations for rocks from the north and south of the Pyrenees are compared and then related to the bathymetry of the Bay of Biscay. 